Article

\title{
Contact Time of Double-Droplet Impacting Superhydrophobic Surfaces with Different Macrotextures
}

\author{
Dian-Ji Lin ${ }^{1,2}$, Ling-Zhe Zhang ${ }^{1,2}$, Meng-Chao $\mathbf{Y i}^{1,2}$, Xin Wang ${ }^{1,2}$, Shu-Rong Gao ${ }^{1,2}$, \\ Yan-Ru Yang ${ }^{1,2}$, Shao-Fei Zheng ${ }^{1,2} \mathbb{D}$ and Xiao-Dong Wang 1,2,* \\ 1 Research Center of Engineering Thermophysics, North China Electric Power University, Beijing 102206, \\ China; victorlin@ncepu.edu.cn (D.-J.L.); zhanglingzhe@ncepu.edu.cn (L.-Z.Z.); \\ yimengchao@ncepu.edu.cn (M.-C.Y.); wangxinlove7@163.com (X.W.); gaoshurong@ncepu.edu.cn (S.-R.G.); \\ cathyyang888@gmail.com (Y.-R.Y.); shaofeizheng56@gmail.com (S.-F.Z.) \\ 2 State Key Laboratory of Alternate Electrical Power System with Renewable Energy Sources, \\ North China Electric Power University, Beijing 102206, China \\ * Correspondence: wangxd99@gmail.com; Tel.: +86-010-6232-1277
}

Received: 14 June 2020; Accepted: 24 July 2020; Published: 26 July 2020

\begin{abstract}
The contact time of droplets on superhydrophobic surfaces is an especially important parameter in many applications, such as self-cleaning, anti-icing, and spray cooling. In this study, we investigate the contact time of two identical droplets simultaneously impacting superhydrophobic surfaces decorated with three different macrotextures, i.e., bathtub-like groove (S1), vertical wall (S2), and rectangular ridge (S3), via lattice Boltzmann method (LBM) simulations. We explore influences of the geometrical parameters of the macrotextures, as well as the center-to-center distance of the two droplets, on the contact time. We found a new rebounding regime with significantly reduced contact times. We demonstrate that, as compared with impacting a smooth superhydrophobic surface, the contact time can be decreased by $41 \%$ for macrotexture $S 1$ because of the asymmetric spreading and retraction of droplets motivated by the macrotexture. We also demonstrate that the new regime depends on the center-to-center distance and geometrical parameters of the macrotextures.
\end{abstract}

Keywords: contact time; double-droplet; macrotexture; impact; superhydrophobic surface

\section{Introduction}

Over the last two decades, superhydrophobic surfaces have gained much attention because of their unique properties, such as strong water repellency, low-contact angle hysteresis, and small friction. After impacting a flat superhydrophobic surface, a droplet undergoes axisymmetric spreading and retraction and eventually bounces off the surface. The duration of time for the droplet contacting with the superhydrophobic surface is defined as contact time, $t_{c}$, which is found to be independent of the Weber number ( $W e=\rho D_{0} v_{0}^{2} / \sigma$ ) in a wide range of impact velocities for low-viscosity fluids [1], where $\rho$ is the liquid density, $D_{0}$ is the initial droplet diameter, $v_{0}$ is the impact velocity, and $\sigma$ is the liquid surface tension. The contact time $t_{\mathrm{c}}$ is usually nondimensionalized by the capillary-inertial time as $t_{\mathrm{c}}^{*}=t_{\mathrm{c}} /\left(\rho D_{0}^{3} / 8 \sigma\right)^{0.5}$.

A shorter contact time is required for various applications like anticorrosion, anti-icing, and antifouling [2-5]. Reducing the contact time has recently become an active field, and various methods have been proposed [6-8]. Superhydrophobic surfaces are usually prepared by patterning various micro/nanotextures on smooth surfaces. Due to axisymmetric spreading and retraction dynamics, the value of $t_{\mathrm{c}}{ }^{*}$ ranges from 2.5 to 2.6 for a water droplet impacting a flat superhydrophobic surface [1], implying that there is a minimum contact time. For the first time, Bird et al. [9] patterned a macro-ridge 
on hydrophobic surfaces to trigger an asymmetric spreading and retraction dynamics in the directions perpendicular and parallel to the ridge. After impacting the ridge, a droplet was split by the ridge into two satellite droplets that rebounded from the surface separately, leading to about $37 \%$ reduction in the contact time. Inspired by this idea, Gauthier et al. [10] experimentally investigated the influences of the impact velocity, droplet radius, and ridge size on the contact time. Their results showed that there were three plateaus of the contact time depending on the impact velocity, and the maximum reduction in the contact time was $44 \%$ in the high-impact velocity regime. Shen et al. [11] conducted a similar experiment but increased the number of macro-ridges from four and five up to eight. They found that a limited overall contact time was close to the time required for spreading to the maximum diameter. Using a lattice Boltzmann method, Lin et al. [12] simulated a droplet impacting a rectangular macro-ridge, and a $48 \%$ reduction in the contact time was achieved. By placing a semi-cylinder on a superhydrophobic surface, Liu et al. [13] reported about $40 \%$ reduction in the contact time when a droplet impacted the semi-cylinder. The reduction was attributed to the fact that the droplet bounced off the semi-cylinder in an elongated shape along the azimuthal direction because of the asymmetric spreading and retraction in the azimuthal and axial directions. In addition, a droplet impacting a concave surface was also investigated in reference [13]. In contrast to impacting the convex surface, the droplet detached from the surface in a shape elongated along the axial direction. Shen et al. [14] released a droplet above dome convex superhydrophobic surfaces, triggering an annulus spreading film. The inner and outer rims of the annulus film retracted with a higher velocity, so that a $28.5 \%$ reduction in the contact time was observed. Chantelot et al. [15] experimentally and numerically studied a droplet impacting a point-like macrotexture. The thin central spreading film was punctured by the singularity, yielding ring bouncing, which resulted in a 52\% reduction in the contact time. Furthermore, a groove was also tested in the Leidenfrost situation. The impacting droplet was elongated along the groove and rapidly took off as a liquid cylinder. A droplet impacting a bathtub-like macrotexture was experimentally studied by Regulagadda et al. [16]. The flow asymmetry was triggered by lamella deflection so as to reduce the contact time. The contact time was found to decrease with an increase in the impact velocity or the inclination of the sloping surface, whereas to increase when a large width of flat region was employed.

It should be noted that the previous studies were mainly concerned about the effects of macrotextures on single-droplet impacts [9-16]; however, multi-droplet impacts are more frequently encountered in applications. When multiple droplets impact a surface, the complex interactions between them may change the dynamic behaviors of the droplets, resulting in different morphologies, as compared with the single-droplet impacts $[17,18]$. Various collision regimes have been observed for double-droplet impacts, such as coalescence, coalescence followed by separation, retarded coalescence, and even bounce-back $[19,20]$. Besides those factors affecting the single droplet impacts, there are more factors that influence the collision regimes of the double-droplet impacts, including impact time difference, center-to-center distance, head-on collision, or off-center collision. Therefore, it is of great importance to study the influences of macrotextures on the dynamic behaviors and contact time of multiple-droplet impacts. So far, such influences are taken into account only for coalescence-induced droplet self-jumping to enhance the jumping velocity. In such a subject, energy conversion efficiency is a key parameter, which is defined as the ratio of the jumping kinetic energy to the released surface energy. Yuan et al. [21] decorated superhydrophobic surfaces with a vertical solid wall as the macrotexture. Their results showed that not only the energy conversion efficiency was increased, but also the jumping direction of the coalesced droplet was altered, which were attributed to the interaction between the liquid bridge and macrotexture. Lu et al. [22] experimentally studied influences of an asymmetric V-groove on coalescence-induced droplet self-jumping. They claimed that their energy conversion efficiency was the highest reported so far. For coalescence-induced droplet self-jumping, two stationary droplets coalesce and bounce from the surface. However, when two droplets impact a surface, the droplets possess a large initial kinetic energy, resulting in a more drastic spreading and retraction process, so that the coalescence and bouncing behaviors differ significantly from two stationary droplets. 
Our recent study [23] showed that there were three kinds of rebound behaviors for two droplets simultaneously impacting a smooth superhydrophobic surface depending on the center-to-center distance between the droplets: complete-coalescence rebound (CCR), partial-coalescence rebound (PCR), and no-coalescence rebound (NCR). The contact time in the PCR regime is significantly smaller than those in the CCR and PCR regimes.

In the present study, a double-droplet impacting superhydrophobic surfaces decorated with different macrotextures is studied via a lattice Boltzmann method. Three kinds of macrotextures are selected, i.e., bathtub-like groove (S1), vertical wall (S2), and single rectangular ridge (S3). The three geometries are selected here because all of them can motivate the asymmetry of the two droplets, thereby promoting droplet rebounding. Influences of the geometrical parameters of the macrotextures, as well as the distance between centers of the two droplets on the contact time, are investigated, and the corresponding mechanisms behind reductions in the contact time are revealed.

\section{Mathematical Model}

\subsection{Shan-Chen Lattice Boltzmann Model}

The lattice Boltzmann method exhibits many advantages in parallelism, no manual tracing interface, and dealing with complex boundary conditions, and hence, it has become a powerful numerical tool. While this method still has some drawbacks, such as a spurious velocity, poor stability for high velocities, and limited density ratio. To alleviate these drawbacks, in this study, a MRT (multiple-relaxation-time) collision operator [24,25], an improved forcing scheme proposed by Li et al. [26], and the Carnahan-Starling (C-S) equation of state [27] are utilized. Here, a brief description about a nineteen-velocity (D3Q19) Shan-Chen model with the MRT (multiple-relaxation-time) collision operator is given, which behaved with a good performance for the simulation of droplet dynamic behaviors [28-30] and is described in our previous articles [12,31].

In the MRT frame, the expression of the evolution equation is [24,25]:

$$
f_{\alpha}\left(\mathbf{x}+\mathbf{e}_{\alpha} \delta_{t}, t+\delta_{t}\right)=f_{\alpha}(\mathbf{x}, t)-\left(\mathbf{M}^{-1} \boldsymbol{\Lambda} \mathbf{M}\right)_{\alpha \beta}\left[f_{\beta}(\mathbf{x}, t)-f_{\beta}^{\mathrm{eq}}(\mathbf{x}, t)\right]+\delta_{t} F_{\alpha}^{\prime}(\mathbf{x}, t)
$$

where $f_{\alpha}(\mathbf{x}, t)$ is the density distribution at position $\mathbf{x}$ and moment $t, \mathbf{e}_{\alpha}$ is the discrete lattice velocity along the $\alpha$ th direction, $\delta_{t}$ is the time step, $\Lambda$ is a diagonal matrix, and $F^{\prime}{ }_{\alpha}$ is the forcing term in the discrete velocity space.

By introducing a matrix, $\mathbf{M}$, the collision process in the right-hand side of Equation (1) can be converted to:

$$
\mathbf{m}^{*}=\mathbf{m}-\boldsymbol{\Lambda}\left(\mathbf{m}-\mathbf{m}^{\mathrm{eq}}\right)+\delta_{t}\left(\mathbf{I}-\frac{\boldsymbol{\Lambda}}{2}\right) \mathbf{S}
$$

where $\mathbf{m}=\mathbf{M f}, \mathbf{m}^{\mathrm{eq}}=\mathbf{M} \mathbf{f}^{\mathrm{eq}}$, and $\mathbf{I}$ represents the identity tensor. The diagonal matrix $\boldsymbol{\Lambda}$ includes the relaxation time:

$$
\boldsymbol{\Lambda}=\operatorname{diag}\left(s_{1}, s_{2}, s_{3}, s_{4}, s_{5}, s_{6}, s_{7}, s_{8}, s_{9}, s_{10}, s_{11}, s_{12}, s_{13}, s_{14}, s_{15}, s_{16}, s_{17}, s_{18}, s_{19}\right)
$$

where $s_{2}$ is related to the bulk viscosity, and $s_{12}=s_{14}=s_{15}=s_{16}$ are related to the shear viscosity. The variable $\mathbf{S}$ denotes the forcing term in the moment space and is expressed as [26]:

$$
\begin{aligned}
& \mathbf{S}=\left(0,38\left(u_{x} F_{x}+u_{y} F_{y}+u_{z} F_{z}\right)+\frac{114 \varepsilon\left(F_{x}^{2}+F_{y}^{2}+F_{z}^{2}\right)}{\psi^{2} \delta_{t}\left(1 / s_{2}-0.5\right)},-11\left(u_{x} F_{x}+u_{y} F_{y}+u_{z} F_{z}\right),\right. \\
& F_{x},-\frac{2}{3} F_{x}, F_{y},-\frac{2}{3} F_{y}, F_{z},-\frac{2}{3} F_{z}, 4 u_{x} F_{x}-2 u_{y} F_{y}-2 u_{z} F_{z},-2 u_{x} F_{x}+u_{y} F_{y}+u_{z} F_{z}, 2 u_{y} F_{y}-2 u_{z} F_{z}, \\
& \left.-u_{y} F_{y}+u_{z} F_{z}, u_{x} F_{y}+u_{y} F_{x}, u_{y} F_{z}+u_{z} F_{y}, u_{x} F_{z}+u_{z} F_{x}, 0,0,0\right)^{T}
\end{aligned}
$$


where $u_{x}, u_{y}$, and $u_{z}$ represent the fluid velocity components in the $x-, y-$, and $z$ directions, and $\varepsilon$ is used to ensure the mechanical stability. The streaming process is given by:

$$
f_{\alpha}\left(\mathbf{x}+\mathbf{e}_{\alpha} \delta_{t}, t+\delta_{t}\right)=f_{\alpha}^{*}(\mathbf{x}, t)=\mathbf{M}^{-1} \mathbf{m}^{*}
$$

where $\mathbf{M}^{-1}$ is the inverse matrix of $\mathbf{M}$. The macroscopic density and velocity are calculated by:

$$
\rho=\sum_{\alpha=0}^{18} f_{\alpha} ; \rho \mathbf{u}=\sum_{\alpha=0}^{18} \mathbf{e}_{\alpha} f_{\alpha}+\frac{\delta_{t}}{2} \mathbf{F}
$$

where $\mathbf{F}=\left(F_{x}, F_{y}, F_{z}\right)$ is the total force exerted on the system, including the fluid-fluid interaction force $\mathbf{F}_{\mathrm{f}}$, the fluid-solid interaction force $\mathbf{F}_{\mathrm{s}}$, and the body force $\mathbf{F}_{\mathrm{b}}$ [32,33], which can be written as:

$$
\begin{gathered}
\mathbf{F}_{\mathbf{f}}(\mathbf{x})=-G \psi(\mathbf{x}) \sum_{\alpha=0}^{18} w_{\alpha} \psi\left(\mathbf{x}+\mathbf{e}_{\alpha} \delta_{t}\right) \mathbf{e}_{\alpha} \\
\mathbf{F}_{\mathbf{s}}(\mathbf{x})=-G_{a d s} \psi(\mathbf{x}) \sum_{\alpha=0}^{18} w_{\alpha} \psi(\mathbf{x}) s\left(\mathbf{x}+\mathbf{e}_{\alpha} \delta_{t}\right) \mathbf{e}_{\alpha} \\
\mathbf{F}_{\mathbf{b}}(\mathbf{x})=\rho(\mathbf{x}) g
\end{gathered}
$$

where $w_{\alpha}$ is the weight factor, $G$ donates the interaction strength among the fluid, $\psi$ is the pseudopotential, which is related to the equation of the state, $s$ is an indicator function, which equals to 1 or 0 , respectively, for solid nodes or fluid nodes, $G_{\text {ads }}$ is used to adjust the force strength of the fluid-solid, i.e., the intrinsic contact angle, and $g$ represents the gravitational acceleration. The pseudopotential $\psi(\mathbf{x})$ can be expressed as [27]:

$$
\psi(\mathbf{x})=\sqrt{\frac{2\left(p-\rho c_{s}^{2}\right)}{G c_{s}^{2}}}
$$

The Carnahan-Starling (C-S) equation of the state is adopted [27]:

$$
p=\rho R T \frac{1+b \rho / 4+(b \rho / 4)^{2}-(b \rho / 4)^{3}}{(1-b \rho / 4)^{3}}-a \rho^{2}
$$

where $a=0.4963\left(R T_{\mathrm{c}}\right)^{2} / p_{\mathrm{c}}, b=0.18727 R T_{\mathrm{c}} / p_{\mathrm{c}}$, and $T_{\mathrm{c}}$ and $p_{\mathrm{c}}$ are the critical temperature and pressure, respectively. In this paper, $a=0.25, b=4$, and $R=1$ are chosen. The liquid density is $\rho_{1}=0.455 \mathrm{mu} \mathrm{lu}^{-3}$ and the vapor density is $\rho_{\mathrm{g}}=0.000606 \mathrm{mu} \mathrm{lu}{ }^{-3}$, corresponding to $T=0.5 T_{\mathrm{c}}$. The surface tension is $\sigma=0.007698 \mathrm{mu} \mathrm{ts}^{-2}$. Here, $\mathrm{mu}$ denotes the mass unit and ts denotes the time step.

\subsection{Model Validation}

To validate the capability of the lattice Boltzmann scheme used in this study, the dynamic process of a droplet impacting a smooth flat superhydrophobic surface is simulated, and the contact time and the maximum spreading diameter are obtained. The droplet diameter $D_{0}$ is fixed at $60 \mathrm{lu}$ (lattice units), and the intrinsic contact angle of the surface is $163^{\circ}$. As mentioned above, when a droplet impacts a smooth flat superhydrophobic surface, the contact time is independent of We in a wide velocity range [1]. In Figure 1a, the dimensionless contact time $t_{\mathrm{c}}{ }^{*}$ as a function of We is shown. It can be seen that the contact time is indeed independent of We. The value of $t_{\mathrm{c}}{ }^{*}$ is 2.48 (the red, dashed line), which is very close to the coefficient of 2.5 in reference [1] and 2.6 in reference [10]. 
(a)

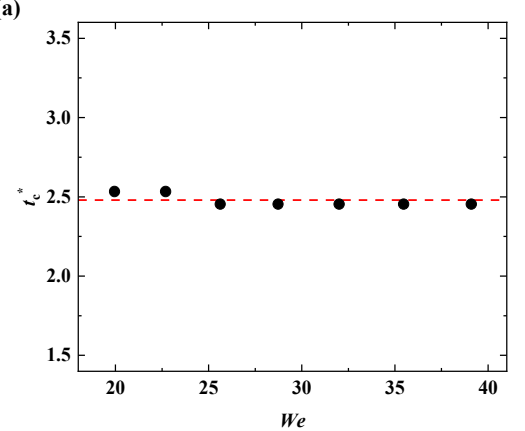

(b)

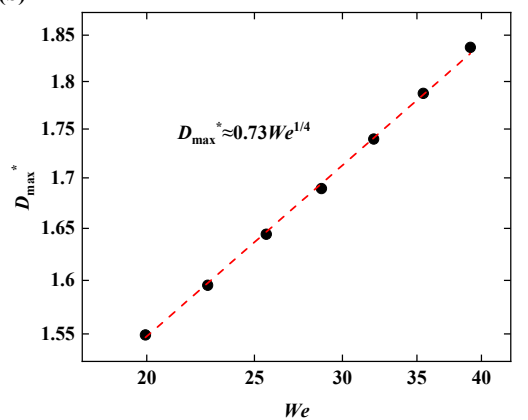

(c)
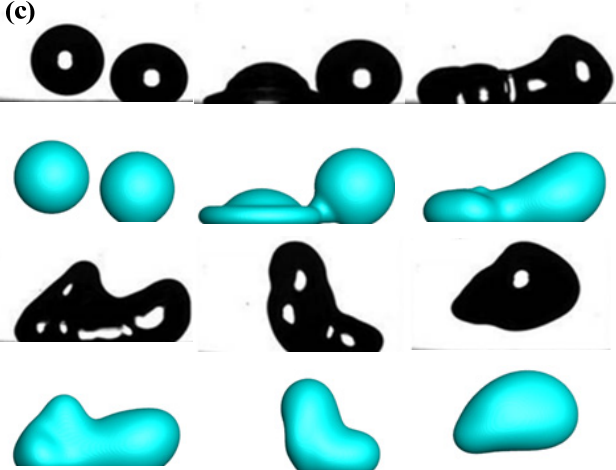

Figure 1. The model validation. (a) The dimensionless contact time as a function of We. (b) The dimensionless maximum spreading diameter as a function of We. (c) The dynamic behavior of a failing droplet impacting a stationary droplet on a superhydrophobic surface with $L_{x}{ }^{*}=1.1$, and $W e=21.8$.

When a droplet of low viscosity impacts a superhydrophobic surface, the maximum-spreading diameter $D_{\max }$ scales as $D_{\max } \sim D_{0} W e^{1 / 4}$ [34]. The maximum spreading diameter $D_{\max }$ can be nondimensionalized as $D_{\max }{ }^{*}=D_{\max } / D_{0}$. As shown in Figure $1 \mathrm{~b}$, the results of $D_{\max }{ }^{*}$ are fitted well by the dashed line, with a slope of $1 / 4$, which are in good agreement with the scaling law. A small deviation is observed at the high We, which may be attributed to a numerical stability for high velocities, but the relative deviation is within $1 \%$, which can be ignored.

There may exist a gas film between the two droplets and between the droplets and the solid surface that play an important role in a double-droplet impacting a superhydrophobic surface. However, the dynamics of the gas film are not observed in our simulations. To verify the interactions between the droplets, the dynamic behavior of a failing droplet impacting a stationary droplet on a superhydrophobic surface is compared with the experiment [35]. The contact angle of superhydrophobic surface is $156^{\circ}$. The distance between the centers of the two droplets in the $x$-direction is defined as $L_{x}$, which can be nondimensionalized as $L_{x}{ }^{*}=L_{x} / D_{0}$. As shown in Figure 1c, the simulated droplet morphologies agree well with the experiment. 


\subsection{Simulation Setup}

Figure 2 shows the schematics of two droplets simultaneously impacting superhydrophobic surfaces decorated with three different macrotextures. In Figure 2a, the first macrotexture looks like a bathtub and has an inclined surface in each side with an angle of inclination $\alpha=45^{\circ}$. The width of the flat valley along the $y$ direction is defined as $L_{y}$. The two droplets are placed above the center of the flat surface. In Figure 2b, the second macrotexture is a vertical solid wall with a height of $h_{1}=70 \mathrm{lu}$, which is mounted on the left side of the two droplets. The height of the vertical solid wall is slightly larger than the droplet diameter, which can guarantee complete contact between the droplets and wall during the spreading process. The distance between the vertical wall and the droplet center along the $y$ direction is defined as $L_{y}$. In Figure 2c, the third macrotexture is a rectangular ridge with a height of $h_{2}$ $=10 \mathrm{lu}$ and a width of $w=2 \mathrm{lu}$, by which a droplet can be split easily [12,31]. Two droplets are located just above the ridge. For the purpose of simplification, the three types of superhydrophobic surfaces shown in Figure 2a-c are defined as S1, S2, and S3, respectively. In all situations, the two droplets have the same diameters of $D_{0}=60 \mathrm{lu}$, and they impact the surface simultaneously with the same velocity $v_{0}$. The Weber number is $W e=\rho D_{0} v_{0}^{2} / \sigma=35.5$, which remains constant in all simulations. The distance between the centers of the two droplets along the $x$-direction is defined as $L_{x}$. The dimensionless factors $L_{x}{ }^{*}$ and $L_{y}{ }^{*}$ are nondimensionalized by $D_{0}$. The parameters used in this study are quantified in terms of the dimensionless parameters; thus, the lu spacing has no effect on the results. The intrinsic contact angles of all solid surfaces are set to be $163^{\circ}$ to ensure surface superhydrophobicity, and the chosen value is based on previous experiments $[10,11,13-15,25]$. No slip boundary condition is applied to all the solid surfaces, and a periodic boundary condition is implemented on the sides of the domain. All the quantities in the simulations are based on the lattice units.
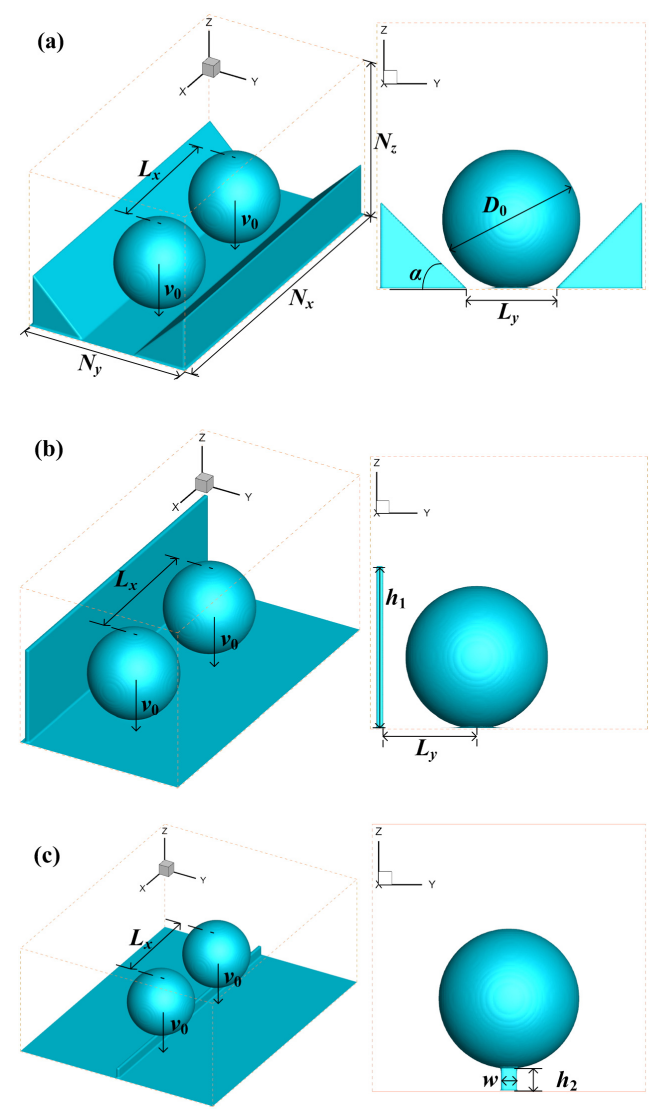

Figure 2. Schematics of two droplets simultaneously impacting superhydrophobic surfaces with three different macrotextures. (a) The S1 surface. (b) The S2 surface. (c) The S3 surface. 


\section{Results and Discussion}

\subsection{Double-Droplet Impact Dynamics}

The morphology evolutions of two droplets simultaneously impacting the superhydrophobic surfaces with three kinds of macrostructures are shown in Figures 3-8, respectively. The initial distances between the centers of the two droplets are set to be $L_{x}^{*}=1.67$ in the three situations.

Figure 3 shows the impact dynamics of the two droplets on the S1 surface at $L_{y}^{*}=0.67$. In the initial stage, the droplets spread freely along the flat valley in the $x$-direction, whereas, in the $y$-direction, the droplets are confined and climb up the inclined surface at both sides. The spherical droplets are surrounded by rims (Figure 3a). The two droplets coalesce rapidly once the rims get in touch with each other. A liquid sheet is formed along the $y$-direction because of the large kinetic energy. Meanwhile, the droplets continue to climb up the inclined surface until they reach the maximum height (Figure 3b). Subsequently, the liquid film on the inclined surface turns to slip down as the coalesced droplet begins to retract in the $y$-direction, which makes the film at the valley get thicker. With the coalesced droplet still spreading along the $x$-direction, the asymmetry between the $x$ and $y$-directions becomes apparent. The liquid sheet raises higher and gets thicker along the $x$-direction (Figure $3 \mathrm{c}, \mathrm{d}$ ). The coalesced droplet reaches its maximum in the $x$-direction when the whole liquid film on the inclined surface almost falls down the valley. Due to the retraction along the $y$-direction, the liquid sheet orientates to the $x$-direction, reaching its largest height approximately (Figure 3e). With the retraction going on, three retraction points located at the center and two sides along the $x$-direction are generated, respectively. More liquid gathers at the retraction points, which promotes the elongated coalesced droplet to form three subunits. All the subunits detach from the surface gradually after the retraction. In the meantime, the coalesced droplet stays in an elongated shape along the $x$-direction (Figure $3 \mathrm{f}-\mathrm{h}$ ).

Figure 4 exhibits the density contours of the coalesced droplet along the center line of the $y$-direction at three specific moments. The liquid bridges between the three subunits become thinner, leading to the formation of two vapor films between the liquid and surfaces. As the vapor films grow continually, the middle subunit bounces off the surface primarily, followed by the subunits at the two sides.

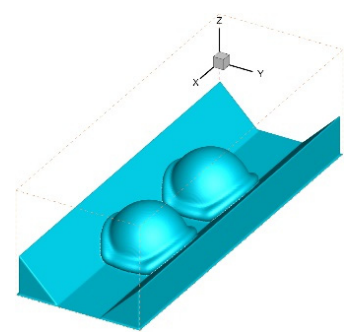

(a) $t^{*}=0.16$

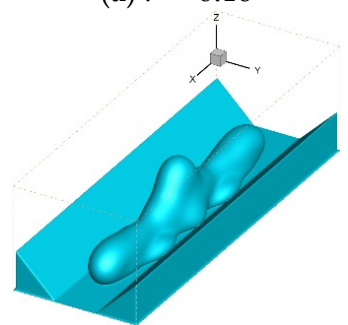

(e) $t^{*}=1.03$

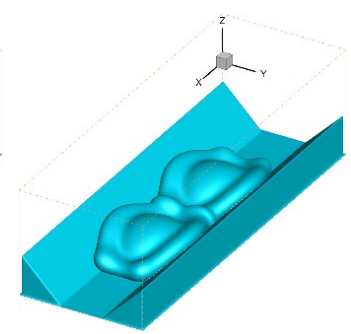

(b) $t^{*}=0.32$

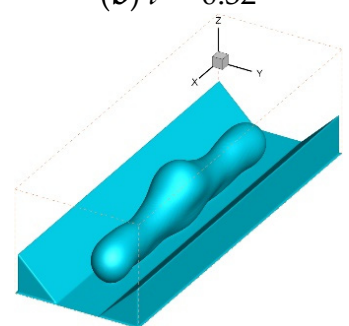

(f) $t^{*}=1.35$

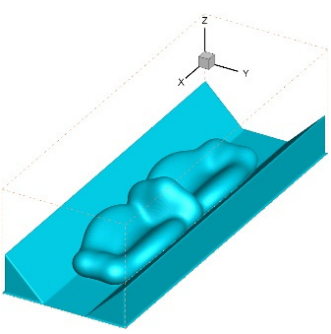

(c) $t^{*}=0.55$

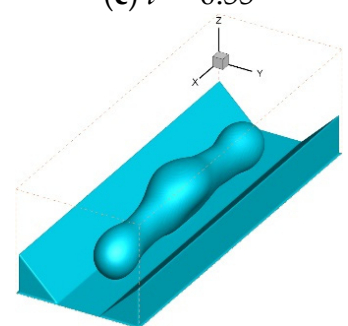

(g) $t^{*}=1.50$

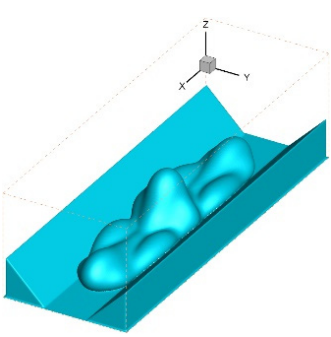

(d) $t^{*}=0.79$

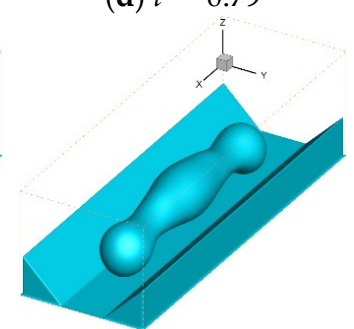

(h) $t^{*}=1.74$

Figure 3. Snapshots of two droplets simultaneously impacting the S1 surface at $L_{x}{ }^{*}=1.67$ and $L_{y}{ }^{*}=$ 0.67. The morphologies at different moments are illustrated in (a-h). 


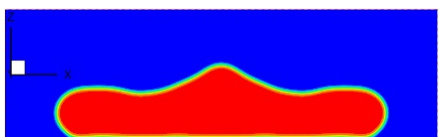

(a) $t^{*}=1.35$

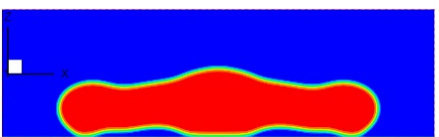

(b) $t^{*}=1.50$

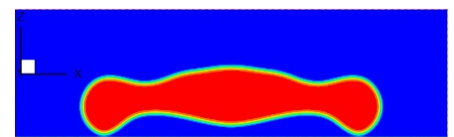

(c) $t^{*}=1.74$

Figure 4. The contours of the coalesced droplet along the center line of the $y$-direction on the S1 surface.

$(\mathbf{a}-\mathbf{c})$ Corresponds to the moments shown in Figure $3 \mathrm{f}-\mathrm{h}$.

The snapshots of two droplets simultaneously impacting the $\mathrm{S} 2$ surface at $L_{y}{ }^{*}=0.58$ are shown in Figure 5. The rims are formed on both the horizontal and vertical surfaces after impact. A significant asymmetry is observed, because the left spreading of the droplets is restricted by the vertical wall, whereas the droplets spread freely at the right side (Figure 5a). A liquid sheet is also generated after the rims meet each other, and the side closest to the vertical wall is higher. Meanwhile, the rims on the vertical wall disappear gradually, but the rims on the horizontal surface become thicker. When the coalesced droplet reaches the maximum, extending along the $y$-direction, it is still spreading in the $x$-direction (Figure $5 b, c)$. Then, the coalesced droplet turns to recoil after finishing the spreading along the $x$-direction. Accompanied with the recoiling in the $y$-direction, the coalesced droplet moves away from the vertical wall gradually. The liquid sheet becomes thicker in the $x$-direction as it keeps falling down. The coalesced droplet is in an elongated shape, which shows a different asymmetry between the $x$ and $y$-directions (Figure $5 \mathrm{~d}$,e). As the recoiling process continues, the coalesced droplet raises up and forms two retraction points in the $x$-direction. Thereafter, two bulges are observed and keep lifting up due to the gathering liquid around the retraction points. As a consequence, the coalesced droplet bounces off the surface eventually (Figure $5 \mathrm{f}-\mathrm{h}$ ).

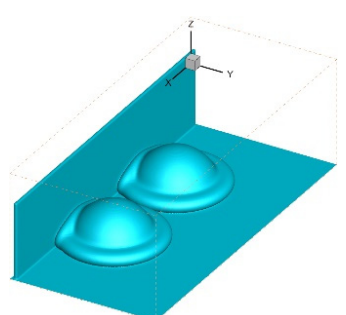

(a) $t^{*}=0.24$

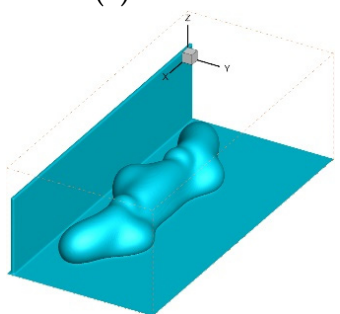

(e) $t^{*}=1.27$

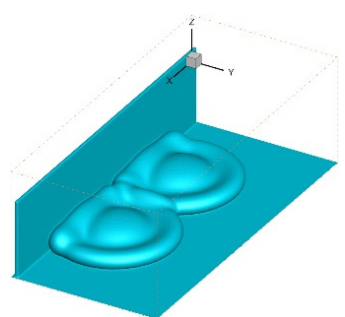

(b) $t^{*}=0.4$

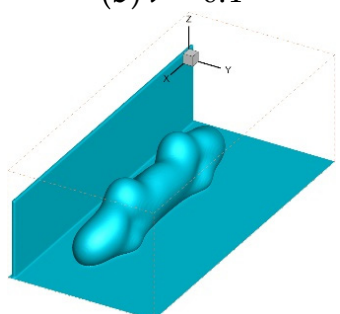

(f) $t^{*}=1.50$

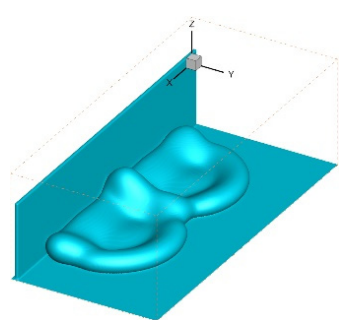

(c) $t^{*}=0.63$

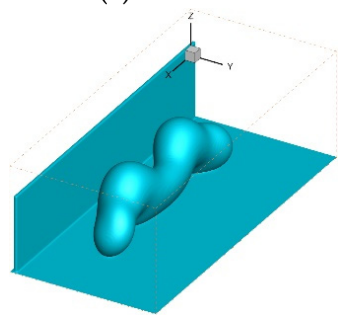

(g) $t^{*}=1.82$

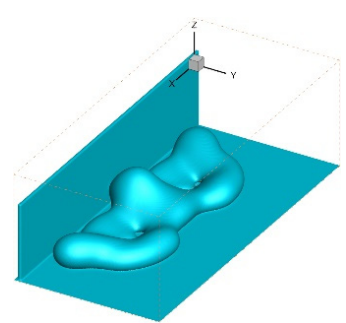

(d) $t^{*}=0.95$

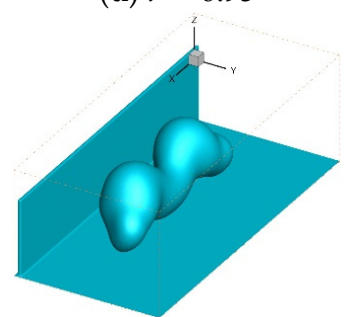

(h) $t^{*}=2.06$

Figure 5. Snapshots of two droplets simultaneously impacting the S2 surface at $L_{x}{ }^{*}=1.67$ and $L_{y}{ }^{*}=$ 0.58 . The morphologies at different moments are illustrated in (a)-(h).

Figure 6 presents the side view of the coalesced droplet at three specific moments. When the coalesced droplet recoils along the $x$-direction, the bulges rise, and the corresponding regions of the coalesced droplet start to depart from the surface, resulting in the appearance of vapor films. Three contact positions are formed between the coalesced droplet and surface. As the bulges keep lifting up, the contact area declines, and the vapor films grow up concomitantly. In the end, the coalesced droplet bounces off the surface as a shape of " $\mathrm{M}$ ".

Figure 7 shows the snapshots of two droplets simultaneously impacting the S3 Surface. Before getting in touch with each other, the two droplets spread on the surface separately and freely at first; then, rims are formed in their periphery. Owing to the asymmetry caused by the ridge, the spreading 
of the droplets on the ridge is slower than that on the flat surface (Figure 7a). Subsequently, the two colliding rims on the flat surface develop into a connecting liquid bridge instead of a liquid sheet. Since the spreading on the flat surface is not restricted, no accumulation of liquid takes place on this occasion. When the liquid on the ridge reaches the maximum spreading, thorough coalescence between the two droplets still does not happen (Figure $7 \mathrm{~b}$ ). The retraction of the liquid film on the ridge promotes the coalesced droplet splitting into two fragments. In the meantime, each fragment keeps spreading on the flat surface until reaching the maximum (Figure $7 \mathrm{c}, \mathrm{d}$ ). Then, two fragments start to retract separately and raise up gradually. The morphologies of the two fragments are similar to those in Figure 5f, i.e., the two fragments are elongated along the $x$-direction, leading to an obvious asymmetry between the $x$ and $y$-directions and generating two retraction points and following with two bulges (Figure 7e). Likewise, liquid accumulates at the two retraction points, leading to the rise of the bulges. After that, the droplets take off from the surfaces separately (Figure $7 \mathrm{f}-\mathrm{h}$ ).

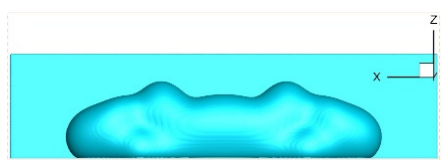

(a) $t^{*}=1.50$

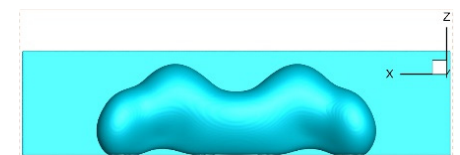

(b) $t^{*}=1.82$

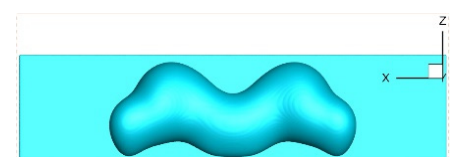

(c) $t^{*}=2.06$

Figure 6. The side view of the coalesced droplet on the S2 surface. $(\mathbf{a}-\mathbf{c})$ Corresponds to the moments shown in Figure $5 \mathrm{f}-\mathrm{h}$.

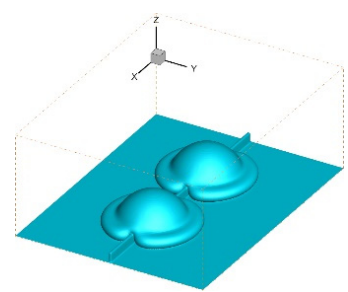

(a) $t^{*}=0.32$ $t_{r}$

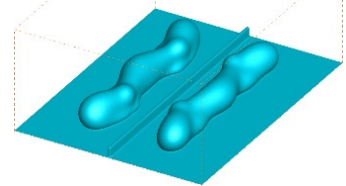

(e) $t^{*}=1.03$

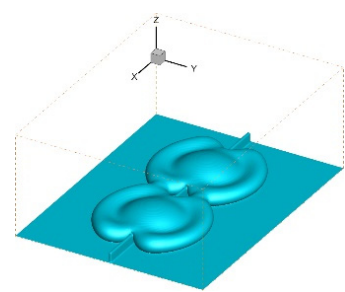

(b) $t^{*}=0.47$ $y_{x}$

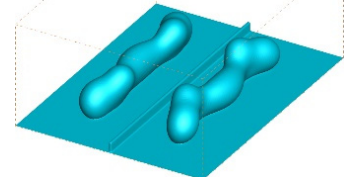

(f) $t^{*}=1.27$

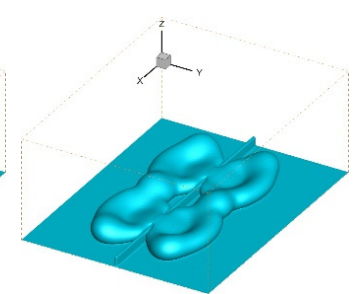

(c) $t^{*}=0.71$ $\mathrm{L}_{\mathrm{x}}^{2}$

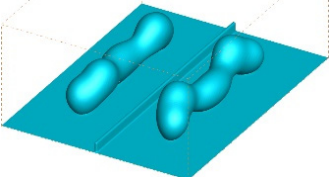

(g) $t^{*}=1.42$

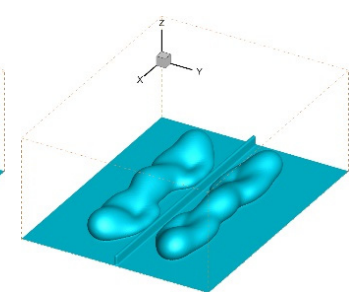

(d) $t^{*}=0.87$ ${ }_{x}^{2}$

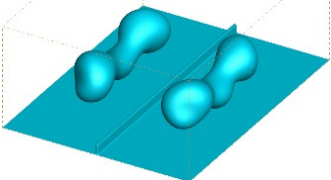

(h) $t^{*}=1.74$

Figure 7. Snapshots of two droplets simultaneously impacting the S3 surface at $L_{x}^{*}=1.67$. The morphologies at different moments are illustrated in $(\mathbf{a}-\mathbf{h})$.

Figure 8 depicts the side view of droplet morphologies at three specific moments. Compared with the droplet morphologies shown in Figure 6, two vapor films are also generated at the positions of the bulges, which gives rise to three contact positions between the droplets and surface, and ultimately, the droplets bounce off the surface as a shape of " $\mathrm{M}$ ". The difference lies in that the retraction points and the bulges are closer to the periphery of the droplets in Figure 8, so that there is more liquid at the ends when the droplets bounce off.

As reported in our recent work [23], when two droplets simultaneously impact a flat superhydrophobic surface, three different rebound behaviors are observed, depending on the distance between the centers of the two droplets, as shown in Figure 9, which are defined as Regime 1, Regime 2, and Regime 3 in this work. Different from the three regimes in Figure 9, the morphologies of the coalesced droplet at the detaching moment in Figure 4, Figure 6, and Figure 8 are similar to the shape of the letter " $\mathrm{M}$ ", and the coalesced droplet has three contact positions with the surface. This new rebound behavior is defined as Regime 4 . 


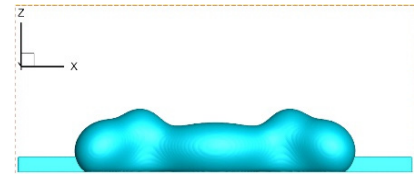

(a) $t^{*}=1.27$

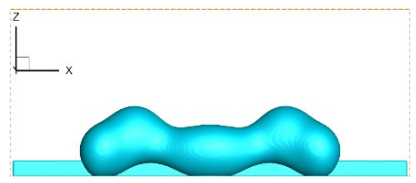

(b) $t^{*}=1.42$

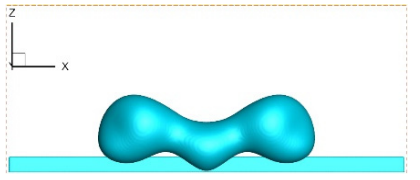

(c) $t^{*}=1.74$

Figure 8. The side view of the coalesced droplet on the S3 surface. (a-c) Corresponds to the moments shown in Figure $7 \mathrm{f}-\mathrm{h}$.

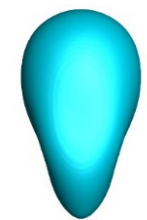

(a) Regime 1

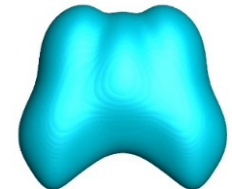

(b) Regime 2

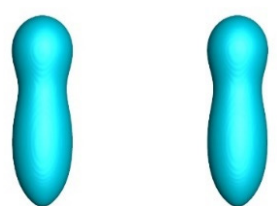

(c) Regime 3

Figure 9. The droplet morphology in each regime. (a) Regime 1. (b) Regime 2. (c) Regime 3.

In the following sections, the rebound regime and contact time as functions of $L_{x}{ }^{*}$ and the geometry parameters of the macrotextures are investigated when two droplets simultaneously impact the S1, S2, and S3 surfaces.

\subsection{Contact Time on the S1 Surface}

Figure 10 presents the contact time as a function of $L_{x}{ }^{*}$ for two droplets simultaneously impacting the $\mathrm{S} 1$ surface with $L_{y}{ }^{*}=0.5,0.67$, or 0.83 . The solid, hollow, cross, and semi-hollow symbols represent Regime 1, Regime 2, Regime 3, and Regime 4, respectively. The minimum of $L_{x}$ is assumed to be $70 \mathrm{lu}$, which is slightly larger than the droplet diameter of $D_{0}=60 \mathrm{lu}$, and hence, the minimum of $L_{x}{ }^{*}$ is $70 / 60=1.167$. The maximum of $L_{x}$ is taken as its upper limit, above which the two droplets cannot coalescence during spreading. Three values of $L_{y}{ }^{*}$ are investigated, and the minimum is equal to the dimensionless droplet radius. As shown in Figure 10, Regime 1 does not occur on the S1 surface, and most of the cases fall into Regime 4. As compared with two droplets impacting a smooth surface at the same $L_{x}{ }^{*}$, the contact time significantly reduces for each $L_{y}{ }^{*}$. For example, for $L_{x}{ }^{*}=1.67$, the contact time reduces by $33 \%$ at $L_{y}{ }^{*}=0.5,30 \%$ at $L_{y}{ }^{*}=0.67$, and $23 \%$ at $L_{y}{ }^{*}=0.83$. The maximum contact time reduction is $41 \%$, which occurs at $L_{x}{ }^{*}=2.0$ and $L_{y}{ }^{*}=0.5$. In a larger range of $L_{x}{ }^{*}=1.5 \sim 2.33$, the contact time can maintain a lower value for each $L_{y}{ }^{*}$. The results can be explained by the fact that the spreading of the droplets is enhanced in the $x$-direction due to the presence of the inclined surface; thus, the two droplets can still coalesce, even though $L_{x}{ }^{*}$ is large, which facilitates the droplet rebounding and makes the rebounding fall into Regime 4, not in Regime 3. For the S1 surface with $L_{y}{ }^{*}$ $=0.5,0.67$, and 0.83 , the critical $L_{x}{ }^{*}$, above which the droplets turn to rebound separately, are 2.67, 2.33, and 2.17, respectively. In other words, the critical $L_{x}{ }^{*}$ declines as the $L_{y}{ }^{*}$ increases.

It is worth noting that the minimum contact time occurs in the range of $L_{x}^{*}=1.5 \sim 2.17$ and decreases with the reduction in $L_{y}{ }^{*}$. To analyze the reasons, the height and spreading diameter of the liquid sheet are defined as $h_{\mathrm{s}}$ and $D_{\mathrm{s}, y}$, as depicted in Figure 11a. The dimensionless height $\left(h_{\mathrm{s}}{ }^{*}\right.$ $\left.=h_{\mathrm{s}} / D_{0}\right)$ and diameter $\left(D_{\mathrm{s}, y}{ }^{*}=D_{\mathrm{s}, y} / D_{0}\right)$ of the liquid sheet at $L_{x}{ }^{*}=1.67$ as a function of time are plotted in Figure 11b,c. As shown in Figure 11b, the dimensionless height $h_{\mathrm{s}}{ }^{*}$ decreases with the $L_{y}{ }^{*}$. Increasing the $L_{y}{ }^{*}$ makes the width of the flat valley larger, so that the spreading in the $y$-direction is enhanced, weakening the spreading in the $x$-direction and the asymmetry between differences in the $x$ and $y$-directions. Thus, when the distance between the centers of the two droplets keep the same values, less liquid prefers to spread in the $x$-direction for the cases with larger $L_{y}{ }^{*}$, leading to a lower maximum height of the liquid sheet. As seen from Figure 11c, there are two reasons for the contact time increasing with the $L_{y}{ }^{*}$. First, the coalescence moment is delayed because of the longer time duration of $D_{\mathrm{s}, y^{*}}=0$, and thus, the contact time increases. Second, the maximum $h_{\mathrm{s}}{ }^{*}$ declines, which 
results in an increase of the $D_{\mathrm{s}, y^{*}}$, whereas the three curves have almost the same retraction velocities and, hence, almost the same retraction times. As results of the two factors, the contact time increases.

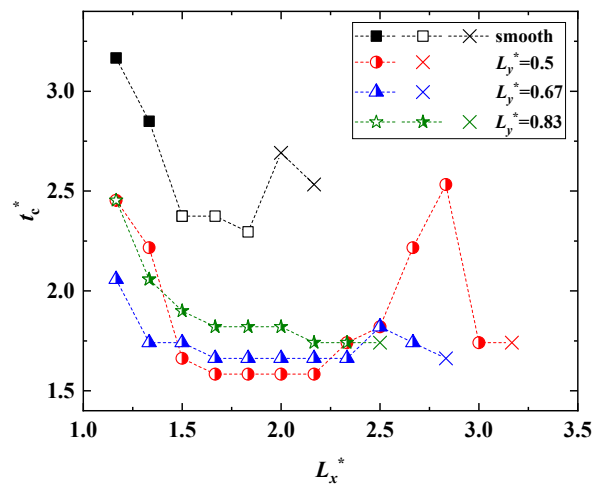

Figure 10. The variations of the contact time with $L_{x}{ }^{*}$ at various $L_{y}^{*}$ on the S1 surface.

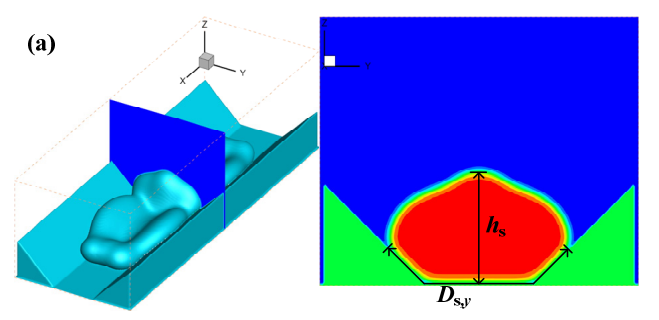

(b)

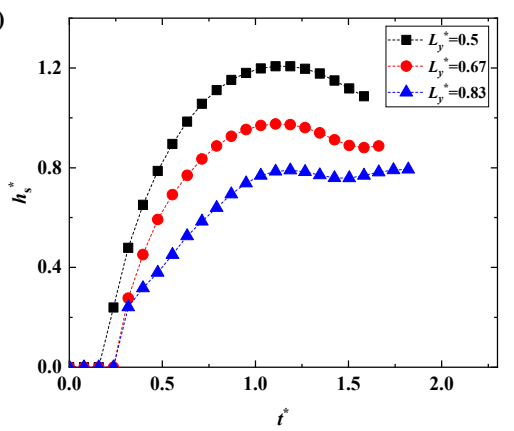

(c)

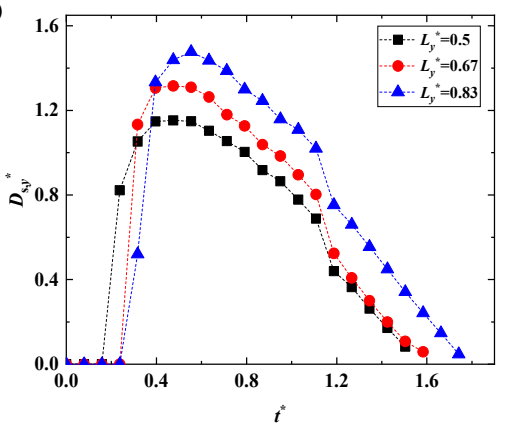

Figure 11. (a) The depiction of $h_{\mathrm{s}}$ and $D_{\mathrm{s}, y}$ of the sheet. The evolutions of (b) $h_{\mathrm{s}}{ }^{*}$ and (c) $D_{\mathrm{s}, y}{ }^{*}$ for various $L_{y}^{*}$ at $L_{x}{ }^{*}=1.67$.

As shown in Figure 10, by comparing the three $L_{y}{ }^{*}$, it is found that the minimum contact time is the highest at $L_{y}{ }^{*}=0.83$, whereas it is the lowest at $L_{y}{ }^{*}=0.5$. When $L_{x}{ }^{*}<1.5$, the contact time at $L_{y}^{*}=$ 0.5 is close to that at $L_{y}{ }^{*}=0.83$ but is longer than that at $L_{y}{ }^{*}=0.67$. When $L_{x}{ }^{*}>2.5$, a drastic fluctuation of the contact time is observed at $L_{y}^{*}=0.5$, where the maximum contact time is nearly equal to that of 
two droplets impacting a flat surface. On the basis of the results above, $L_{y}{ }^{*}=0.67$ is a better choice, because it achieves a relatively low and stable contact time.

Figure 12 compares the effects of the distance between the two droplets $\left(L_{x}^{*}\right)$ on the morphologies of the coalesced droplet. The morphologies are observed at the departure moment. As the $L_{x}{ }^{*}$ increases, the coalesced droplet exhibits a more uniform mass distribution, and more subunits are observed. For example, when the $L_{x}{ }^{*}$ increases from 1.33 to 2.67 , the number of subunits increases from 3 to 5 .

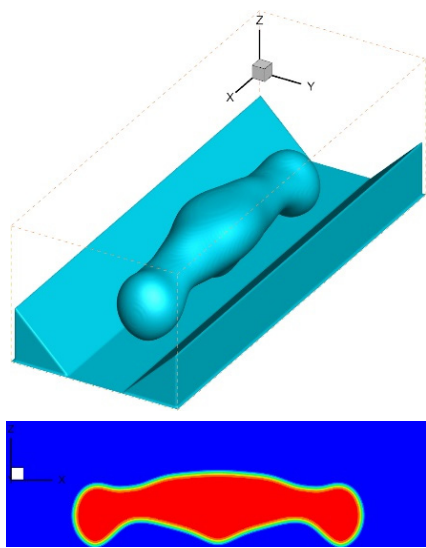

(a) $L x^{*}=1.33$

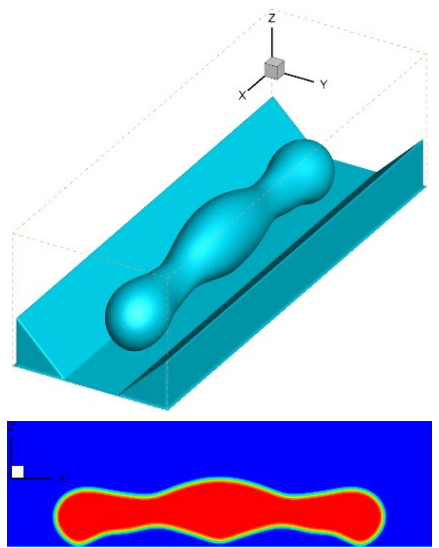

(b) $L x^{*}=2.0$

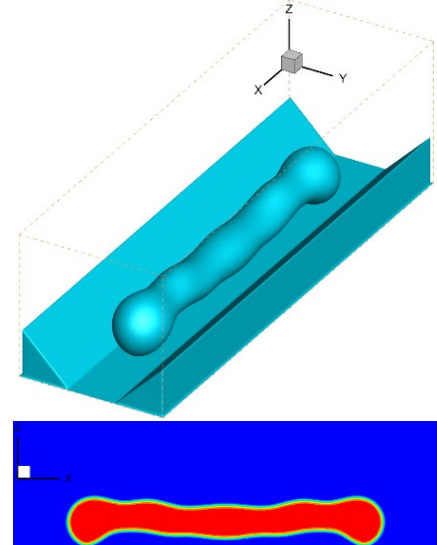

(c) $L x^{*}=2.67$

Figure 12. The morphologies of the droplets at various $L_{x}^{*}$ on the S1 surface with $L_{y}^{*}=0.67$. (a) $L_{x}{ }^{*}=1.33$. (b) $L_{x}{ }^{*}=2.0$. (c) $L_{x}{ }^{*}=2.67$.

\subsection{Contact Time on the S2 Surface}

The contact time as a function of $L_{x}{ }^{*}$ for two droplets simultaneously impacting the S2 surface with three specific $L_{y}{ }^{*}$ is presented in Figure 13. When $L_{x}{ }^{*}<1.5$, except for the two cases with $L_{y}{ }^{*}=0.5$ and $L_{x}{ }^{*}=1.33$, as well as $L_{y}{ }^{*}=0.5$ and $L_{x}{ }^{*}=1.33$, rebounding is in Regime 1 , and the contact time is larger than that on a smooth surface. When $L_{x}^{*}>2.0$, rebounding is in Regime 3 or 4 , and the contact time increases with the $L_{x}{ }^{*}$ and gradually approaches to that on the smooth surface. However, a special case is observed at $L_{y}{ }^{*}=0.5$ and $L_{x}{ }^{*}=2.5$, in which the contact time even exceeds that on a smooth surface. When $1.5 \leq L_{x}{ }^{*} \leq 2.0$, the S2 surface shows its advantages. Rebounding falls into Regime 4, and the contact time is remarkably lower than that on a smooth surface. For example, as compared with a smooth surface, the contact time is reduced by $21 \%$ at $L_{x}{ }^{*}=1.67$ for all three $L_{y}{ }^{*}$.

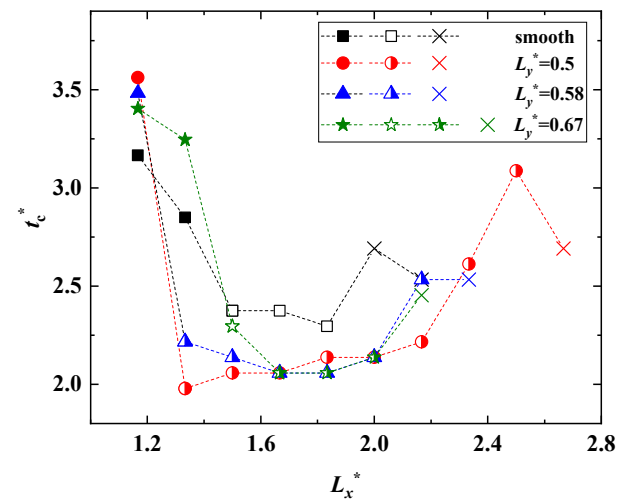

Figure 13. The variations of the contact times with $L_{x}{ }^{*}$ at various $L_{y}{ }^{*}$ on the S2 surface.

For the S2 surface, the macrotexture is a vertical wall that is mounted on the left side of the droplets. As a result, spreading and retraction of the droplets are asymmetric along the $y$-direction. Once the coalesced droplet touches the vertical wall, a force will be exerted on the coalesced droplet, leading 
to a rightward velocity component. Thus, when the coalesced droplet bounces off the surface, the jumping velocity is no longer perpendicular to the surface. Figure 14a shows the droplet morphology after the coalesced droplet bounces off the surface for a short period of time. The red dotted line represents the initial position of the mass center of the two droplets in the $y$-direction, and the black dotted line represents the present position of the mass center. The offset length is defined as $p_{y}$, and the dimensionless offset length is defined as $p_{y}{ }^{*}=p_{y} / D_{0}$. Figure $14 \mathrm{~b}$ presents $p_{y}{ }^{*}$ as a function of time at a fixed $L_{x}{ }^{*}=1.67$. Here, the distances between the vertical wall and droplets are taken as $L_{y}{ }^{*}=0.5$, 0.58 , and 0.67 , respectively. For all three $L_{y}{ }^{*}$, the $p_{y}{ }^{*}$ continuously increase with time, indicating that the coalesced droplet is away from the vertical wall. When the initial position of the two droplets is closer to the vertical wall, i.e., the $L_{y}{ }^{*}$ is smaller, and the $p_{y}{ }^{*}$ increases faster. In other words, the coalesced droplet moves away from the vertical wall more quickly. This is because the rim and sheet of the droplets touch the vertical wall earlier for a smaller $L_{y}{ }^{*}$, leading to a stronger force acting on the coalesced droplet and, hence, a larger rightward velocity component. Figure $14 \mathrm{c}$ shows $p_{y}{ }^{*}$ as a function of time at a fixed $L_{y}{ }^{*}=0.58$. Here, the distances between the centers of the two droplets are chosen as $L_{x}{ }^{*}=1.33,1.67$, and 2.0. A smaller $L_{x}{ }^{*}$ makes the $p_{y}{ }^{*}$ increase faster. This result can be explained as follows. For a smaller $L_{x}^{*}$, the two droplets come into contact with each other earlier, so that the coalesced droplet possesses a larger kinetic energy. As a result, the coalesced droplet impacts the vertical wall more violently, and hence, the wall exerts a stronger counterforce on the coalesced droplet.

(a)

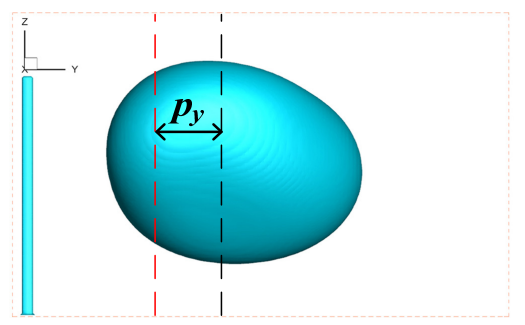

(b)

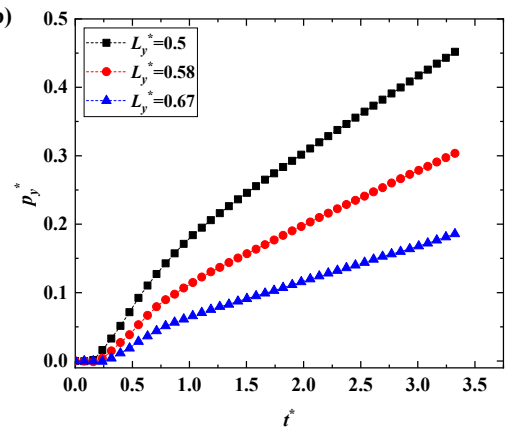

(c)

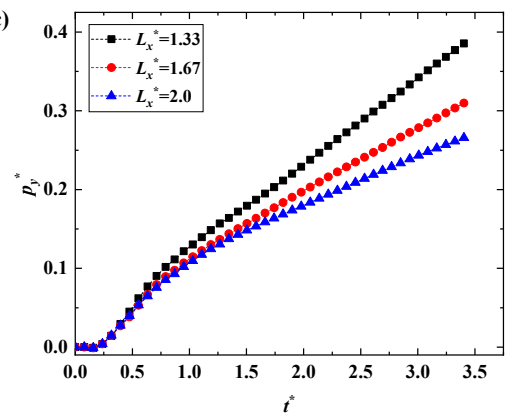

Figure 14. (a) The droplet morphology after bouncing off the S2 surface. (b) The dimensionless $p_{y}{ }^{*}$ as a function of time at a fixed $L_{x}{ }^{*}=1.67$. (c) The dimensionless $p_{y}{ }^{*}$ as a function of time at a fixed $L_{y}{ }^{*}=0.58$. 


\subsection{Contact Time on the S3 Surface}

The influence of $L_{x}{ }^{*}$ on the contact time for two droplets simultaneously impacting the S3 surface is presented in Figure 15. The rebounding mainly falls into Regime 4, so that the contact time is reduced significantly, as compared with that on a smooth surface. The minimum contact time occurs at $L_{x}{ }^{*}=1.5$, which is $33 \%$ lower than that on a smooth surface. Due to the presence of the ridge, the coalesced droplet is split into two fragments, which move away from each other during retraction and bounce off the surface separately. Interestingly, the jumping directions of the two fragments are not perpendicular to the surface, because the fragments have a horizontal velocity component. An offset length, $p_{y}$, is employed to characterize the magnitude of the horizontal velocity component, as shown in Figure 16a, and it is scaled with the droplet diameter as $p_{y}{ }^{*}=p_{y} / D_{0}$. The dimensionless offset length, $p_{y}{ }^{*}$, as a function of time is shown in Figure 16b. Here, the dimensionless distance between the centers of the two droplets is fixed as $L_{x}{ }^{*}=1.67$. It can be seen that $p_{y}{ }^{*}$ vs. $t^{*}$ is approximately linear, and hence, the component of the jumping velocity in the $y$-direction remains constant.

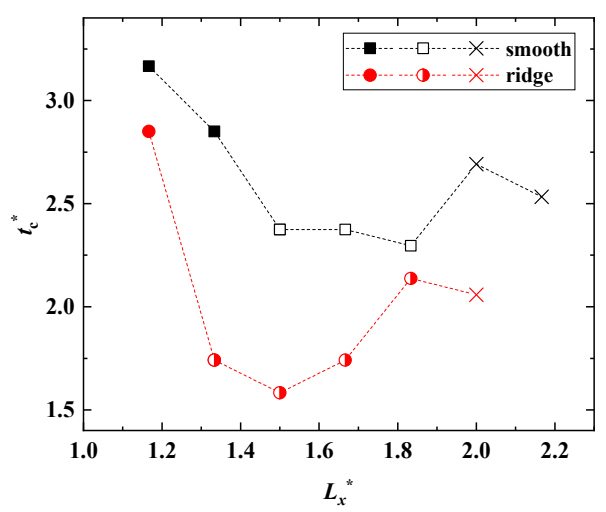

Figure 15. The variations of the contact time with $L_{x}{ }^{*}$ on the S3 surface.

(a)

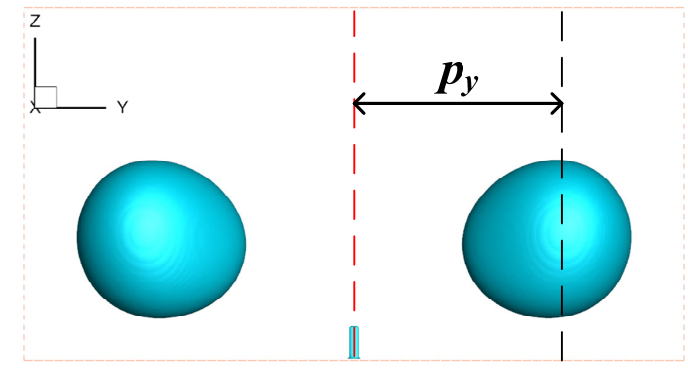

(b)

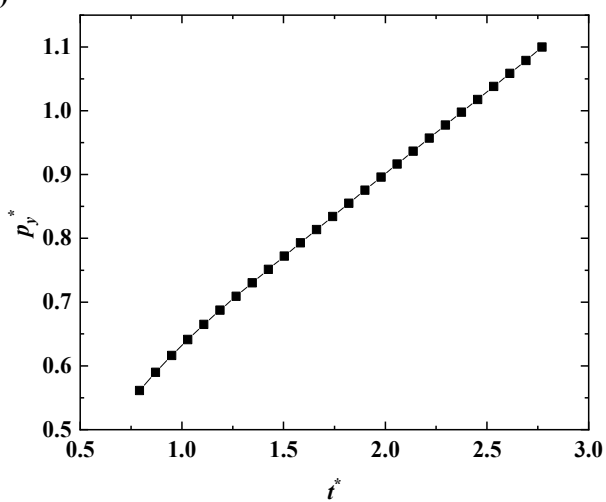

Figure 16. (a) The droplet morphology after bouncing off the S3 surface. (b) The dimensionless $p_{y}{ }^{*}$ as a function of time at a fixed $L_{x}{ }^{*}=1.67$. 
Influences of the three macrotextures, i.e., S1, S2, and S3, on the contact time of two droplets impacting simultaneously are compared in Figure 17. The contact times for all the three macrotextures are lower than that on a smooth superhydrophobic surface. The main differences among them lie in the following three aspects. First, the smallest contact time occurs on the S1 surface, followed by the S2 and S3 surfaces. Second, the smallest contact time on the S1 surface can be maintained in a wider $L_{x}{ }^{*}$ range, whereas this range is the narrowest on the S3 surface. Third, the S2 and S3 surfaces are able to alter the jumping direction of the coalesced droplet, even if for center impact. These findings provide useful guidance for designing hydrophobic surfaces for anti-icing and self-cleaning applications.

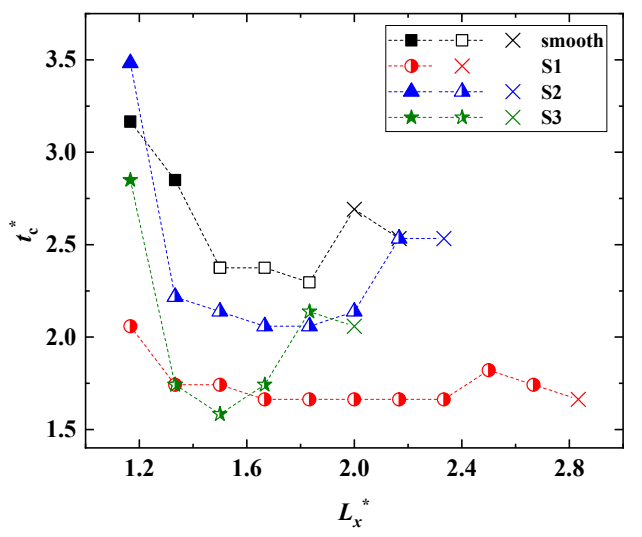

Figure 17. Comparison of contact times on the S1, S2, and S3 surfaces. Here, $L_{y}{ }^{*}=0.67$ on the S1 surface, and $L_{y}{ }^{*}=0.58$ on the S2 surface.

\section{Conclusions}

In this study, the dynamic behaviors of double droplets simultaneously impacting superhydrophobic surfaces with three different macrotextures, i.e., bathtub-like groove (S1), vertical wall (S2), and single rectangular ridge (S3), are investigated using a lattice Boltzmann method. The effects of geometrical parameters of the macrotextures and the distances between the centers of the two droplets on the contact time are analyzed. A new rebounding regime is found, in which the coalesced droplet forms three contact positions with the surfaces during retraction and, finally, leaves the surfaces in a shape of " $\mathrm{M}$ ", reducing the contact time significantly. In contrast to the contact time of double droplets simultaneously impacting a smooth superhydrophobic surface, the maximum reduction in the contact time is $41 \%$. For all the three macrotextures, the contact time reaches the minimum when the dimensionless distance of the centers between the two droplets meets $L_{x}{ }^{*}=1.5 \sim 2.17$. On the S1 surface, the width of the flat valley significantly influences the coalescence dynamics of the two droplets, and a small width promotes coalescence, leading to a lower minimum contact time. The ability to reduce the contact time is the weakest for the S2 surface, but S2 can change the jumping direction of the coalesced droplet, making the droplet bounce off the surface obliquely. Reducing the distance between the two droplets and S2, or reducing the distance between the centers of the two droplets, causes a stronger counterforce exerted by S2 on the coalesced droplet, and hence, the jumping deviates from the vertical direction more violently. When impacting the S3 surface, the droplets are split by S3, and the split fragments have a horizontal velocity component. As a result, the fragments can also bounce off the surface obliquely. Further research on this subject should consider the effects of impact velocity, successive impact, and off-center impact. Moreover, an array of macrotextures is also worth studying because of its practical importance.

Author Contributions: Conceptualization, D.-J.L.; data curation, L.-Z.Z., X.W., S.-R.G., and Y.-R.Y.; formal analysis, X.W.; funding acquisition, X.-D.W.; investigation, D.-J.L.; methodology, D.-J.L., M.-C.Y., and S.-R.G.; project administration, X.-D.W.; resources, S.-R.G. and Y.-R.Y.; software, L.-Z.Z., M.-C.Y., X.W., and Y.-R.Y.; supervision, X.-D.W.; validation, S.-F.Z.; visualization, S.-F.Z.; writing — original draft, D.-J.L.; and writing-review and editing, L.-Z.Z., M.-C.Y., S.-F.Z., and X.-D.W. All authors have read and agreed to the published version of the manuscript. 
Funding: This research was partially funded by the State Key Program of National Natural Science of China (No. 51936004), the National Science Fund for Distinguished Young Scholars of China (No. 51525602), the Science Fund for Creative Research Groups of the National Natural Science Foundation of China (No. 51821004), and the Fundamental Research Funds for the Central Universities (No. 2020MS060).

Conflicts of Interest: The authors declare no conflict of interest.

\section{References}

1. Richard, D.; Clanet, C.; Quéré, D. Surface phenomena: Contact time of a bouncing drop. Nature 2002, 417, 811. [CrossRef] [PubMed]

2. $\quad$ Lin, D.-J.; Zhang, L.-Z.; Yi, M.-C.; Wang, X.; Gao, S.-R.; Yang, Y.-R.; Wang, X.-D. Rebound Dynamics of Two Droplets Successively Impacting an Inclined Surface. Coatings 2020, 10, 592. [CrossRef]

3. Tembely, M.; Vadillo, D.; Soucemarianadin, A.; Dolatabadi, A. Numerical Simulations of Polymer Solution Droplet Impact on Surfaces of Different Wettabilities. Processes 2019, 7, 798. [CrossRef]

4. Xie, F.-F.; Lv, S.-H.; Yang, Y.-R.; Wang, X.-D. Contact Time of a Bouncing Nanodroplet. J. Phys. Chem. Lett. 2020, 11, 2818-2823. [CrossRef]

5. Zhang, Z.; Ge, B.; Men, X.; Li, Y. Mechanically durable, superhydrophobic coatings prepared by dual-layer method for anti-corrosion and self-cleaning. Colloids Surf. A Physicochem. Eng. Asp. 2016, 490, $182-188$. [CrossRef]

6. Shen, Y.; Tao, H.; Chen, S.-L.; Xie, Y.; Zhou, T.; Wang, T.; Tao, J. Water repellency of hierarchical superhydrophobic Ti6Al4V surfaces improved by secondary nanostructures. Appl. Surf. Sci. 2014, 321, 469-474. [CrossRef]

7. Tsai, P.A.; Pacheco, S.; Pirat, C.; Lefferts, L.; Lohse, D. Drop Impact upon Micro- and Nanostructured Superhydrophobic Surfaces. Langmuir 2009, 25, 12293-12298. [CrossRef]

8. Lv, C.; Hao, P.; Zhang, X.; He, F. Drop impact upon superhydrophobic surfaces with regular and hierarchical roughness. Appl. Phys. Lett. 2016, 108, 141602. [CrossRef]

9. Bird, J.C.; Dhiman, R.; Kwon, H.-M.; Varanasi, K.K. Reducing the contact time of a bouncing drop. Nature 2013, 503, 385-388. [CrossRef]

10. Gauthier, A.; Symon, S.; Clanet, C.; Quéré, D. Water impacting on superhydrophobic macrotextures. Nat. Commun. 2015, 6, 8001. [CrossRef]

11. Shen, Y.; Tao, J.; Tao, H.; Chen, S.; Pan, L.; Wang, T. Approaching the theoretical contact time of a bouncing droplet on the rational macrostructured superhydrophobic surfaces. Appl. Phys. Lett. 2015, 107, 111604. [CrossRef]

12. Lin, D.-J.; Wang, L.; Wang, X.-D.; Yan, W.-M. Reduction in the contact time of impacting droplets by decorating a rectangular ridge on superhydrophobic surfaces. Int. J. Heat Mass Transf. 2019, 132, 1105-1115. [CrossRef]

13. Liu, Y.; Andrew, M.; Li, J.; Yeomans, J.M.; Wang, Z. Symmetry breaking in drop bouncing on curved surfaces. Nat. Commun. 2015, 6, 10034. [CrossRef]

14. Shen, Y.; Liu, S.; Zhu, C.; Tao, J.; Chen, Z.; Tao, H.; Pan, L.; Wang, G.; Wang, T. Bouncing dynamics of impact droplets on the convex superhydrophobic surfaces. Appl. Phys. Lett. 2017, 110, 221601. [CrossRef]

15. Chantelot, P.; Moqaddam, A.M.; Gauthier, A.; Chikatamarla, S.S.; Clanet, C.; Karlin, I.V.; Quéré, D. Water ring-bouncing on repellent singularities. Soft Matter 2018, 14, 2227-2233. [CrossRef] [PubMed]

16. Regulagadda, K.; Bakshi, S.; Das, S.K. Triggering of flow asymmetry by anisotropic deflection of lamella during the impact of a drop onto superhydrophobic surfaces. Phys. Fluids 2018, 30, 072105. [CrossRef]

17. Zhou, W. Lattice Boltzmann simulation of coalescence of multiple droplets on nonideal surfaces. Phys. Rev. E 2015, 92, 053307. [CrossRef]

18. Raman, K.A.; Jaiman, R.K.; Lee, T.-S.; Low, H.-T. Dynamics of simultaneously impinging drops on a dry surface: Role of impact velocity and air inertia. J. Colloid Interface Sci. 2017, 486, 265-276. [CrossRef]

19. Qian, J.; Law, C.K. Regimes of coalescence and separation in droplet collision. J. Fluid Mech. 1997, 331, 59-80. [CrossRef]

20. Amani, A.; Balcázar, N.; Gutiérrez, E.; Oliva, A. Numerical study of binary droplets collision in the main collision regimes. Chem. Eng. J. 2019, 370, 477-498. [CrossRef]

21. Yuan, Z.; Hu, Z.; Chu, F.; Wu, X. Enhanced and guided self-propelled jumping on the superhydrophobic surfaces with macrotexture. Appl. Phys. Lett. 2019, 115, 163701. [CrossRef] 
22. Lu, D.; Zhao, M.; Zhang, H.; Yang, Y.; Zheng, Y. Self-Enhancement of Coalescence-Induced Droplet Jumping on Superhydrophobic Surfaces with an Asymmetric V-Groove. Langmuir 2020, 36, 5444-5453. [CrossRef]

23. Wang, X.; Lin, D.-J.; Wang, Y.-B.; Gao, S.-R.; Yang, Y.-R.; Wang, X.-D. Rebound dynamics of two droplets simultaneously impacting a flat superhydrophobic surface. AIChE J. 2020, e16647. [CrossRef]

24. Zheng, S.; Eimann, F.; Philipp, C.; Fieback, T.; Gross, U. Single droplet condensation in presence of non-condensable gas by a multi-component multi-phase thermal lattice Boltzmann model. Int. J. Heat Mass Transf. 2019, 139, 254-268. [CrossRef]

25. Zheng, S.; Eimann, F.; Fieback, T.; Xie, G.; Gross, U. Numerical investigation of convective dropwise condensation flow by a hybrid thermal lattice Boltzmann method. Appl. Therm. Eng. 2018, 145, 590-602. [CrossRef]

26. Li, Q.; Luo, K.H.; Li, X.J. Lattice Boltzmann modeling of multiphase flows at large density ratio with an improved pseudopotential model. Phys. Rev. E 2013, 87, 053301. [CrossRef]

27. Yuan, P.; Schaefer, L. Equations of state in a lattice Boltzmann model. Phys. Fluids 2006, 18, 42101. [CrossRef]

28. Zhang, D.; Papadikis, K.; Gu, S. Three-dimensional multi-relaxation time lattice-Boltzmann model for the drop impact on a dry surface at large density ratio. Int. J. Multiph. Flow 2014, 64, 11-18. [CrossRef]

29. Zhang, D.; Papadikis, K.; Gu, S. Application of a high density ratio lattice-Boltzmann model for the droplet impingement on flat and spherical surfaces. Int. J. Therm. Sci. 2014, 84, 75-85. [CrossRef]

30. Shi, Y.; Tang, G.; Xia, H. Investigation of coalescence-induced droplet jumping on superhydrophobic surfaces and liquid condensate adhesion on slit and plain fins. Int. J. Heat Mass Transf. 2015, 88, 445-455. [CrossRef]

31. Lin, D.J.; Zhang, L.Z.; Yi, M.C.; Gao, S.R.; Yang, Y.R.; Wang, X.D. Contact time on inclined superhydrophobic surfaces decorated with parallel macro-ridges. Colloids Surf. A Physicochem. Eng. Asp. 2020, 599, 124924. [CrossRef]

32. Yu, Y.; Li, Q.; Zhou, C.Q.; Zhou, P.; Yan, H. Investigation of droplet evaporation on heterogeneous surfaces using a three-dimensional thermal multiphase lattice Boltzmann model. Appl. Therm. Eng. 2017, 127, 1346-1354. [CrossRef]

33. Zhang, L.-Z.; Yuan, W.-Z. A lattice Boltzmann simulation of coalescence-induced droplet jumping on superhydrophobic surfaces with randomly distributed structures. Appl. Surf. Sci. 2018, 436, 172-182. [CrossRef]

34. Clanet, C.; Béguin, C.; Richard, D.; Quéré, D. Maximal deformation of an impacting drop. J. Fluid Mech. 2004, 517, 199-208. [CrossRef]

35. Farhangi, M.M.; Graham, P.J.; Choudhury, N.R.; Dolatabadi, A. Induced Detachment of Coalescing Droplets on Superhydrophobic Surfaces. Langmuir 2012, 28, 1290-1303. [CrossRef] [PubMed] 